\title{
Optimization of Molecularly Imprinted Polymer Method for Rapid Screening of $17 \beta$-Estradiol in Water by Fluorescence Quenching
}

\author{
Yu Yang and Edward P. C. Lai \\ Department of Chemistry, Carleton University, Ottawa, ON, Canada K1S $5 B 6$ \\ Correspondence should be addressed to Edward P. C. Lai, edward_lai@carleton.ca \\ Received 21 January 2011; Revised 3 May 2011; Accepted 16 May 2011 \\ Academic Editor: Stig Pedersen-Bjergaard
}

Copyright ( $) 2011$ Y. Yang and E. P. C. Lai. This is an open access article distributed under the Creative Commons Attribution License, which permits unrestricted use, distribution, and reproduction in any medium, provided the original work is properly cited.

A new method was optimized for rapid screening of $17 \beta$-estradiol (E2) in water under 10 min. Molecularly imprinted polymer (MIP) particles $\left(325 \pm 25 \mathrm{~nm}\right.$ ) were added in a water sample at $\mathrm{pH} 5.5$ and $20^{\circ} \mathrm{C}$ to form a suspension. Fluorescence emission from E2 nonspecifically bound onto the MIP particles was first quenched by large gold nanoparticles ( $43 \pm 5 \mathrm{~nm})$. The Stern-Volmer plot was linear, with dynamic quenching constants $\left(K_{\mathrm{sv}}\right)$ of $2.9 \times 10^{4} \mathrm{M}^{-1}$. Fluorescence emission from E2 specifically bound inside the MIP particles was next quenched by small nitrite anions that easily penetrated the imprinted cavities. The Stern-Volmer plot became nonlinear, with $K_{\mathrm{sv}}=2.1 \times 10^{2} \mathrm{M}^{-1}$ and static quenching constant $(\mathrm{V})$ below $1.0 \mathrm{M}^{-1}$. The difference between these two emission intensities varied as the initial E2 concentration in water, generating a Scatchard calibration curve with $R^{2}>0.97$ from 0.1 to $10 \mathrm{ppb}$.

\section{Introduction}

Molecularly imprinted polymers (MIPs) exhibiting high selectivity and affinity to the target molecule are well recognized as a fast growing research field $[1,2]$. They have been successfully applied in various novel methods of chemical analysis [3, 4], including potentiometric sensors [5-7], amperometric detection [8], and differential pulse cathodic stripping voltammetry [9]. An optical sensor was fabricated with an MIP film for the determination of formaldehyde molecules that induced measurable optical reflectivity shifts [10]. For surface plasmon resonance spectroscopy, MIP particles were spin-coated onto a gold surface to detect theophylline [11]. MIP fibers were used in a sensing device to determine folic acid [12].

A novel sensing scheme based on nonlinear fluorescence quenching of $17 \beta$-estradiol (E2) was recently developed in our laboratory [13]. Small nitrite ions penetrated the porous structure of MIP particles and quenched the fluorescence emission from E2 molecules inside imprinted cavities. On the contrary, large methacrylamide molecules (3-D stearic diameter $=0.536 \mathrm{~nm}$ ) were hindered when penetrating the pores to access the imprinted cavities, resulting in low dynamic quenching. Research was continued in our laboratory to evaluate larger quenchers, such as gold nanoparticles (AuNPs) that could readily be synthesized with a diameter of $43 \pm 5 \mathrm{~nm}$ [14-16]. Their effectiveness was studied with regard to quenching the fluorescence of only nonspecifically bound E2 molecules throughout the porous MIP particles, but not those specifically bound inside the imprinted cavities, as illustrated in Scheme 1. The objective of this work was to develop a rapid method (hopefully under $5 \mathrm{~min}$ ) for the determination of trace $\mathrm{E} 2$ in water (down to $0.1 \mathrm{ppb}$ ).

\section{Experimental Section}

2.1. Chemicals. Sodium citrate tribasic dihydrate, gold (III) chloride trihydrate, sodium nitrite, and E2 were purchased from Sigma-Aldrich (St. Louis, MO, USA). Methacrylic acid and methacrylamide were purchased from Aldrich (Milwaukee, WI, USA). 2,2-azobisisobutyro-nitrile (AIBN) was purchased from Pfaltz and Bauer (Waterbury, CT, USA). 


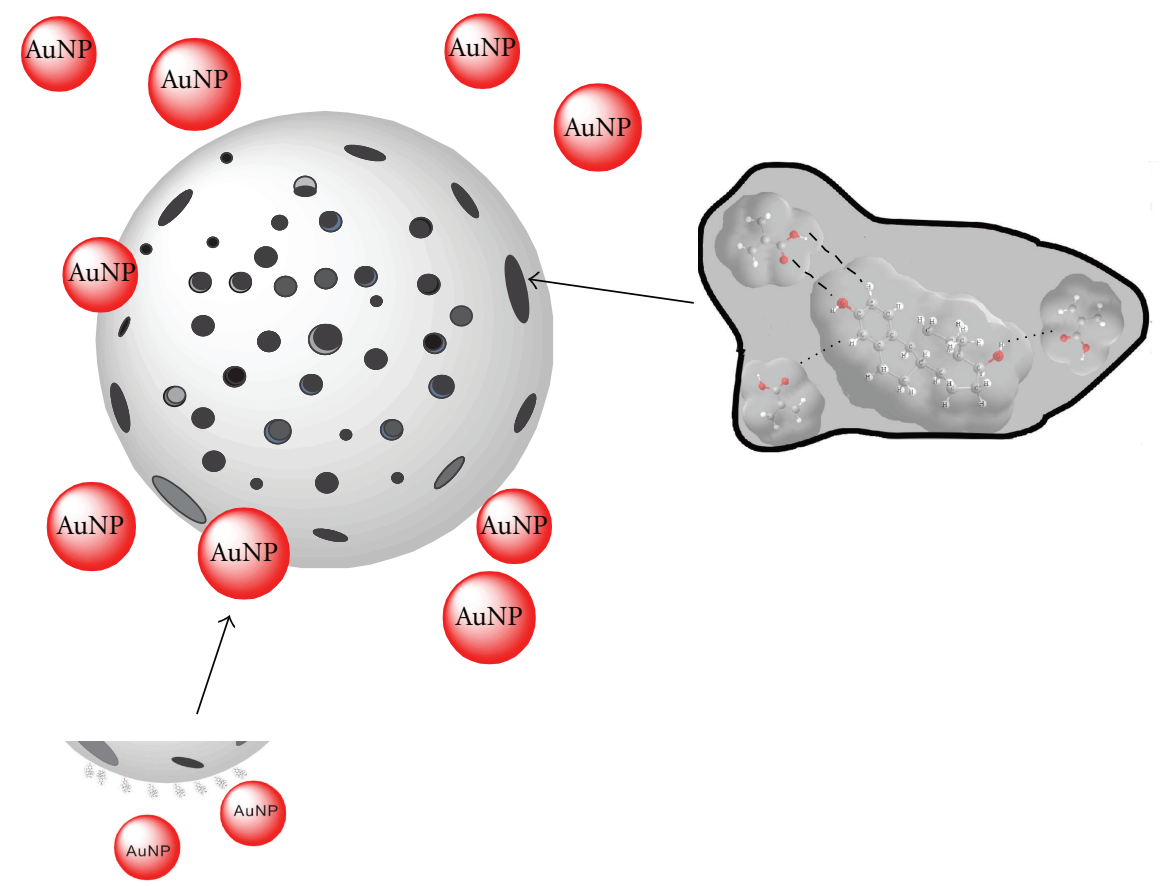

Scheme 1: Larger AuNPs were used in the first step of fluorescence quenching to quench E2 molecules nonspecifically bound throughout the porous MIP particle while small nitrite anions easily penetrate the MIP particle to quench the fluorescence emission from E2 molecules specifically bound inside imprinted cavities in the second step of fluorescence quenching. The attenuated fluorescence emission intensity between step 1 and step 2 varies as a function of E2 concentration.

Methanol (HPLC grade), acetonitrile (HPLC grade), and acetone (spectro grade) were purchased from Caledon (Georgetown, ON, Canada). Acetic acid (reagent grade) was purchased from Anachemia (Montreal, QC, Canada). $18-\mathrm{M} \Omega \cdot \mathrm{cm}$ distilled deionized water $(\mathrm{DDW})$ was obtained from a Millipore Milli-Q water system (Bedford, MD, USA).

2.2. Preparation of MIP Submicron Particles and AuNPs. The method for preparation of E2 MIP submicron particles had previously been described [17]. These particles $(80 \mathrm{~mL})$ were washed with $15 \%$ acetic acid in methanol $(\mathrm{v} / \mathrm{v})$, methanol, acetonitrile, and DDW three times. Each washing was combined with $1 \mathrm{hr}$ of sonication and $1 \mathrm{hr}$ of centrifugation to completely extract template E2 molecules from the particles and remove polymerization reagent residues. After the last washing with DDW, the $\mathrm{pH}$ was $5.5 \pm 0.1$ in the supernatant and the free E2 concentration was below the detection limit of HPLC-FD instrument. These washed MIP submicron particles were dried at $70^{\circ} \mathrm{C}$. Another batch of freshly prepared MIP submicron particles was washed only with DDW for 25 times. These washed E2-MIP particles would contain the maximum E2 loading [13]. AuNPs were synthesized by adapting a previously reported method [18]. No washing was applied to these AuNPs.

2.3. Particle Size Analysis. The AuNPs, MIP, and E2-MIP particles were suspended in $10 \mathrm{M} \mathrm{KNO}_{3}$ at a concentration of $40 \mathrm{mg} / \mathrm{mL}$. The suspensions were sonicated for $15 \mathrm{~min}$ before measurement on a NanoDLS particle size analyzer (Brookhaven Instruments, Holtsville, NY, USA). The instrument had been calibrated by $92 \pm 4 \mathrm{~nm}$ Nanosphere size standards (Duke Scientific, Palo Alto, CA, USA). A total of 10 measurements were run after $30 \mathrm{~s}$ of quiescence time, and the laser beam intensity was automatically optimized by the analyzer before each run.

2.4. Fluorescence Quenching. $3.5 \mathrm{ppm} \mathrm{E} 2(2 \mathrm{~mL})$ and $2.5 \mathrm{mg} /$ $\mathrm{mL}$ E2-MIP particles $(2 \mathrm{~mL})$ were added into two $3-\mathrm{mL}$ quartz cuvette cells, each equipped with a polytetrafluoroethylene (PTFE) stopper. Then $1 \mathrm{~mL}$ of AuNPs aqueous suspension with elemental concentrations from $0 \mathrm{M}$ to 5.88 $\times 10^{-4} \mathrm{M}$ was used to quench the E2 and E2-MIP particles fluorescence emission intensities. All emission intensities were measured at room temperature $\left(20 \pm 1^{\circ} \mathrm{C}\right)$ by a fluorescence spectrophotometer (Varian Cary Eclipse, Palo Alto, CA, USA) using excitation wavelength of $280 \pm 1 \mathrm{~nm}$ and emission wavelength of $310 \pm 1 \mathrm{~nm}$ (or scanning from $290 \mathrm{~nm}$ to $450 \mathrm{~nm}$ ). Both the excitation and emission slits were set at $5 \mathrm{~nm}$. To test for inner filter effect, $1 \mathrm{~mL}$ of $1 \%(\mathrm{w} / \mathrm{w})$ trisodium citrate dihydrate was used instead of AuNPs. Similarly, E2 and E2-MIPs fluorescence quenching experiments with sodium nitrite were accomplished under exactly the same experimental conditions.

Two-step fluorescence quenching by first AuNPs and then sodium nitrite was performed. $7.7 \pm 0.1 \mathrm{mg}$, $5.5 \pm 0.1 \mathrm{mg}, 3.3 \pm 0.1 \mathrm{mg}$, and $1.1 \pm 0.1 \mathrm{mg}$ of templateremoved MIP submicron particles were added into $2.2 \mathrm{~mL}$ of E2 aqueous solution with concentrations from $0.0001 \mathrm{ppm}$ to $3.5 \mathrm{ppm}$. The blank and E2-templated MIP particles were prepared by using the same amount of template-removed 


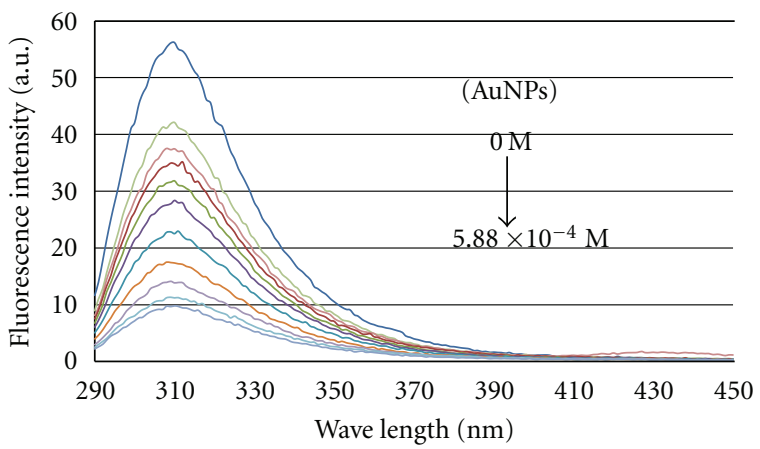

(a)

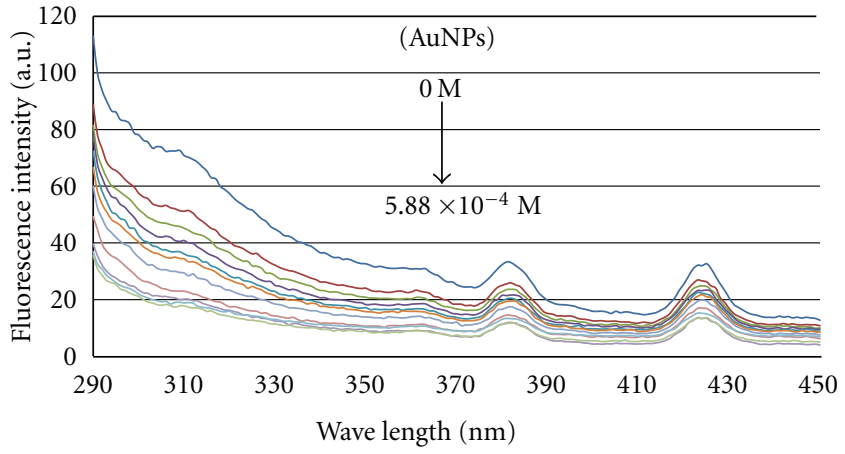

(b)

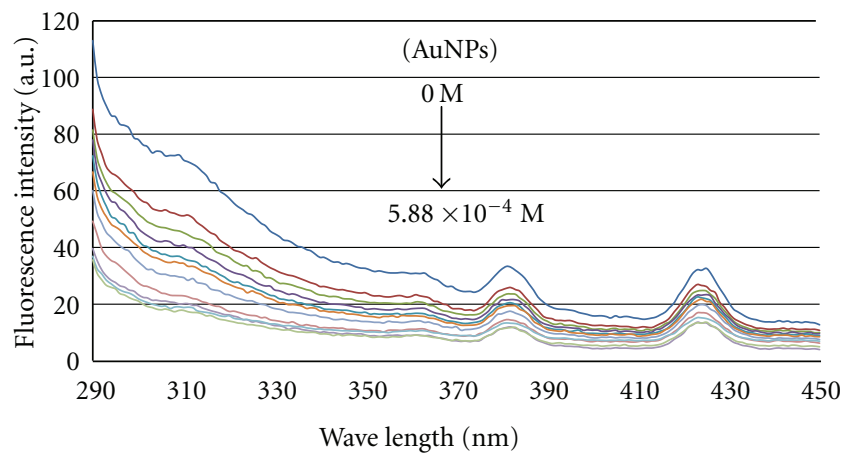

(c)

FIGURE 1: Fluorescence emission spectra of (a) $3.5 \mathrm{ppm}$ E2 aqueous solution, (b) $2.5 \mathrm{mg} / \mathrm{mL}$ E2-MIP particles in aqueous suspension, and (c) $2.5 \mathrm{mg} / \mathrm{mL}$ E2-bound NIP particles in aqueous suspension, during fluorescence quenching by AuNPs from $0 \mathrm{M}$ to $5.88 \times 10^{-4} \mathrm{M}$. $\left(\lambda_{\mathrm{ex}}=\right.$ $280 \mathrm{~nm})$.

MIPs and E2-MIP particles suspended in $2.2 \mathrm{~mL}$ of DDW. All of these suspensions were incubated under sonication for $5-35 \mathrm{~min}$ at room temperature $\left(20 \pm 1^{\circ} \mathrm{C}\right)$. Then, $2.0 \mathrm{~mL}$ of E2-bound MIP or E2-MIP submicron particle suspension was transferred into a $3 \mathrm{~mL}$ quartz cuvette cell and spiked with $1 \mathrm{~mL}$ of $5.88 \times 10^{-4} \mathrm{M}$ AuNPs aqueous suspension. After the fluorescence emission intensity was recorded, $100 \mu \mathrm{L}$ of $150 \pm 1 \mathrm{mg} / \mathrm{mL}$ sodium nitrite was added to perform the second step of fluorescence quenching.

All light absorption spectra by quenchers were measured on a UV-visible spectrophotometer (Varian Cary 3, Palo Alto, CA, USA) by scanning from $250 \mathrm{~nm}$ to $350 \mathrm{~nm}$ at room temperature $\left(20 \pm 1^{\circ} \mathrm{C}\right)$ to investigate the inner filter effect. The absorption of both exciting light $\left(\lambda_{\mathrm{ex}}=280 \pm 1 \mathrm{~nm}\right)$ and fluorescence emission $\left(\lambda_{\mathrm{em}}=310 \pm 1 \mathrm{~nm}\right)$ by quenchers was corrected, for right-angle illumination, as described elsewhere [19].

\section{Results and Discussion}

3.1. Fluorescence Quenching. E2 is a naturally fluorescent compound. After it interacts with nonfluorescent MIP particles both specifically and nonspecifically [20], the bound E2 molecules can be determined by spectrofluorimetry $\left(\lambda_{\mathrm{ex}}=\right.$ $280 \mathrm{~nm}$ and $\lambda_{\mathrm{em}}=310 \mathrm{~nm}$ ) [21]. Figure 1 shows the fluorescence emission spectra of E2, E2-MIP particles, and E2-bound NIP particles during their quenching by AuNPs.
Without particles, a 3.5 ppm E2 aqueous solution exhibited decreasing fluorescence intensities when AuNPs were added stepwise as shown in Figure 1(a). The fluorescence intensity decreased by almost $82 \%$ from the initial level as the concentration of AuNPs reached $5.88 \times 10^{-4} \mathrm{M}$. Similarly, the quenching effects of AuNPs on E2-MIP particles and E2-bound NIP particles are evidenced in Figures 1(b) and 1 (c), decreasing the fluorescence intensity by $76 \%$ and $77 \%$. The strong Mie scattering peak (at $280 \mathrm{~nm}$ ) from particles slightly enhanced all E2 fluorescence emission peaks at $310 \mathrm{~nm}$, which can be considered meritorious for the determination of E2 at trace levels. Two Raman scattering peaks (at $380 \mathrm{~nm}$ and $425 \mathrm{~nm}$ ) were characteristic of particles when an excitation wavelength of $280 \mathrm{~nm}$ was used. Luckily, they did not have any significant impact on the fluorescence quenching results.

The fluorescence properties of AuNPs were studied before they were used as a large fluorescence quencher in all subsequent experiments. When $5.88 \times 10^{-4} \mathrm{M}$ of AuNPs were examined by scanning the excitation wavelength in Figure 2(a) and using an emission wavelength of $310 \mathrm{~nm}$, only one Mie scattering peak was observed at $310 \mathrm{~nm}$. When the emission wavelength was scanned in Figure 2(b) using an excitation wavelength of $280 \mathrm{~nm}$, only two Mie scattering peaks were found at $280 \mathrm{~nm}$ (first order) and $570 \mathrm{~nm}$ (second order). Therefore the AuNPs were nonfluorescent, making them ideal for use as fluorescence quencher in this work. 


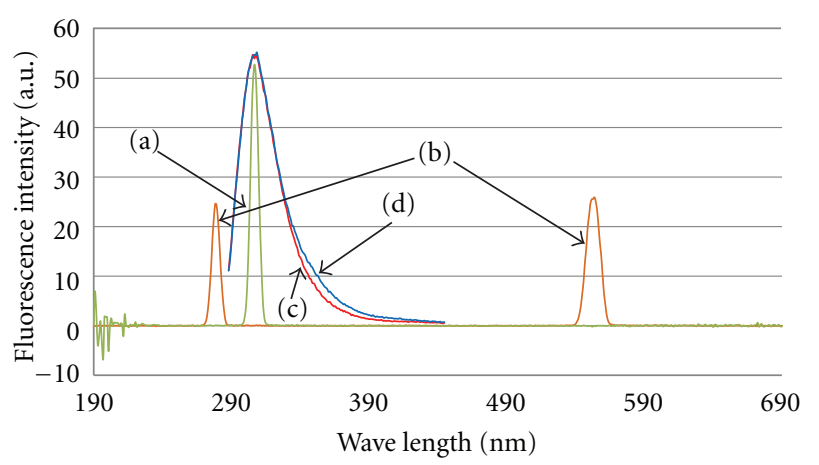

Figure 2: (a) Fluorescence excitation spectrum of AuNPs aqueous suspension $\left(\lambda_{\mathrm{em}}=310 \mathrm{~nm}\right)$, (b) fluorescence emission spectrum (first and second orders) of AuNPs aqueous suspension $\left(\lambda_{\mathrm{ex}}=\right.$ $280 \mathrm{~nm}$ ), (c) fluorescence emission spectrum of E2 aqueous solution $\left(\lambda_{\mathrm{ex}}=280 \mathrm{~nm}\right)$, and (d) fluorescence emission spectrum of E2 aqueous solution in presence of trisodium citrate dihydrate $(1 \% \mathrm{w} / \mathrm{w})\left(\lambda_{\mathrm{ex}}=280 \mathrm{~nm}\right)$.

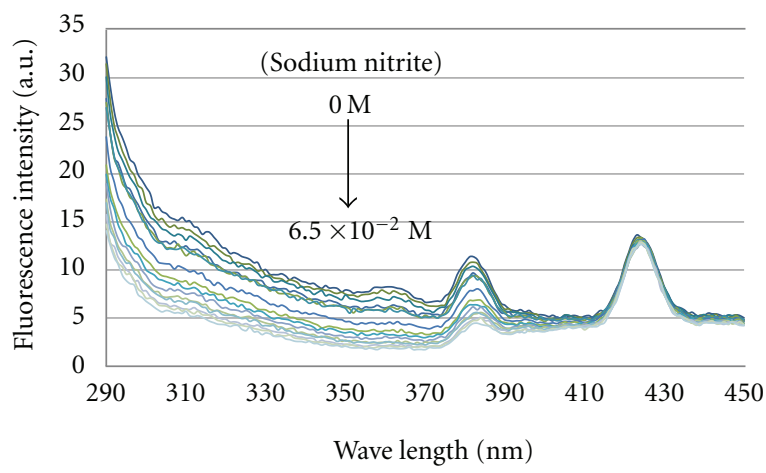

FIGURE 3: Fluorescence emission spectra during titration of sodium nitrite (up to a final concentration of $6.5 \times 10^{-2} \mathrm{M}$ ) into a mixture of $2.5 \mathrm{mg} / \mathrm{mL}$ E2-MIP particles and $5.88 \times 10^{-4} \mathrm{M}$ AuNPs. The titration consisted of fifteen $10-\mu \mathrm{L}$ spikes of $100 \mathrm{mg} / \mathrm{mL}$ sodium nitrite to minimize any dilution effect $(\sim 6 \%)$.

Figure 2(c) shows the fluorescence emission spectrum of 3.5 ppm E2 aqueous solution while Figure 2(d) shows the same spectrum after addition of trisodium citrate dehydrate $(1 \% \mathrm{w} / \mathrm{w})$. No significant inner filter effect was observed from $1 \%$ trisodium citrate dihydrate, which was present in the synthesis of AuNPs. Furthermore in real samples, E2 may exist with some metabolites or other related compounds that can fluorescence. However, no interferences would be possible because these other fluorescent compounds cannot bind with the MIP cavities. Therefore after fluorescence quenching by AuNPs, the interferences can be eliminated.

In our previous study [13], sodium nitrite was able to quench the fluorescence emissions from E2 aqueous solution, E2-MIP aqueous suspension, and E2-bound NIP aqueous suspension. Hence, it was used in this work to finish the fluorescence quenching job after AuNPs quenched only the fluorescence emission from E2 that were nonspecifically bound inside particles. As shown in Figure 3, the residual fluorescence emission from $2.5 \mathrm{mg} / \mathrm{mL}$ E2-MIP particles in

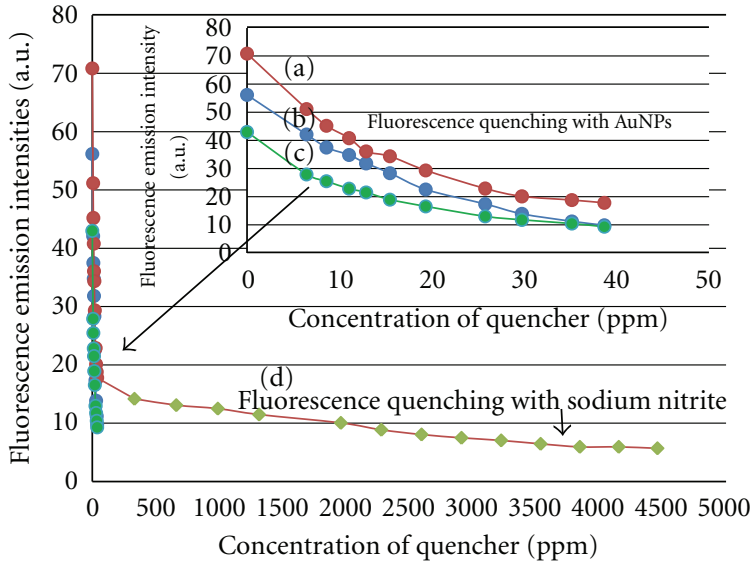

Figure 4: Fluorescence emission intensity at $310 \mathrm{~nm}$ versus concentration of quencher: (a) $2.5 \mathrm{mg} / \mathrm{mL}$ E2-MIP particles in aqueous suspension quenched with AuNPs, (b) 3.5 ppm E2 aqueous solution quenched with AuNPs, (c) $2.5 \mathrm{mg} / \mathrm{mL}$ E2-bound NIP particles in aqueous suspension quenched with AuNPs, (d) $2.5 \mathrm{mg} / \mathrm{mL}$ E2MIP particles in aqueous suspension going through two steps of fluorescence quenching, first with AuNPs and next with sodium nitrite (standard error bars, approximately three-times the size of each data point symbol, are omitted here for the sake of clarity).

aqueous suspension, after quenching with $5.88 \times 10^{-4} \mathrm{M}$ AuNPs, was an intensity of $14.2 \pm 0.2$ arbitrary units (a.u.) coming mostly from E2 specifically bound inside the MIP cavities. Sodium nitrite was then titrated, stepwise from $0 \mathrm{M}$ to $6.5 \times 10^{-2} \mathrm{M}$, into the mixture of E2-MIP particles and AuNPs. Due to its small size, the nitrite anion penetrated through the porous MIP particles and quenched the fluorescence emission from the specifically bound E2 molecules. At the end of titration, the emission intensity was reduced to $5.5 \pm 0.2$ a.u. This result clearly demonstrated how simple it was to determine the amount of specifically bound E2 molecules.

Figure 4 plots all the fluorescence emission intensities measured (at $310 \mathrm{~nm}$ ) from Figures 1 and 3. Intuitively, both free E2 molecules in aqueous solution and nonspecifically bound E2 molecules in NIP particles (which had no imprinted cavities) in aqueous suspension were all accessible by AuNPs to undergo collisional quenching. If there were no imprinted cavities in MIP particles to protect the specifically bound E2 molecules (from quenching by AuNPs), the final emission intensity in Figure 4(a) would probably be as low as the $\sim 10$ a.u. in Figures 4 (b) and 4(c) plots when the AuNPs quencher concentration reached $38.7 \mathrm{ppm}(=5.88$ $\times 10^{-4} \mathrm{M}$ ). In reality, the E2-MIP particles contained some inaccessible E2 molecules that contributed to a higher final emission intensity of $\sim 18$ a.u.

3.2. Quencher Sizes and Efficiencies. The MIP and NIP particles studied in this work had diameters, as measured by a nanoDLS particle size analyzer, of $477 \pm 11 \mathrm{~nm}$ and $373 \pm 21 \mathrm{~nm}$, respectively. E2 molecules were specifically bound inside the MIP cavities that were complementary in size, shape, and arrangement of functional groups. Small 


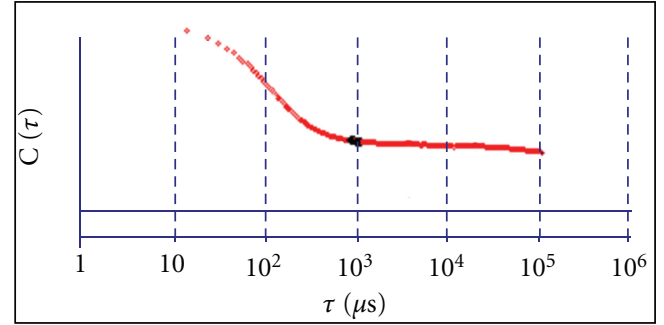

\begin{tabular}{|cccc|}
\hline Run & Eff. diam. $(\mathrm{nm})$ & Half width $(\mathrm{nm})$ & Polydispersity \\
\hline 1 & 39.8 & 22.9 & 0.33 \\
2 & 38 & 21.2 & 0.312 \\
3 & 49 & 28.4 & 0.335 \\
4 & 38.1 & 21.6 & 0.322 \\
5 & 39.4 & 22.5 & 0.327 \\
6 & 42 & 24 & 0.326 \\
7 & 44.5 & 25.1 & 0.318 \\
8 & 45.7 & 25.5 & 0.31 \\
9 & 85 & 47 & 0.305 \\
10 & 33.1 & 17.7 & 0.284 \\
\hline Mean & 45.5 & 25.6 & 0.317 \\
Std. error & 4.6 & 2.5 & 0.005 \\
Combined & 43 & 24.4 & 0.322 \\
\hline
\end{tabular}

FIGURE 5: Correlation of light scattering intensity with time for measuring the size of AuNPs on a nanoDLS particle size analyzer.

nitrite anions could easily penetrate the porous MIP particles and quench the fluorescence from the E2 molecules by dynamic collisions. The large AuNPs used in this study had a diameter of $43 \pm 5 \mathrm{~nm}$. Figure 5 shows the correlation of light scatting intensity with time, as obtained for AuNPs during a particle size measurement. Over 10 runs, the particle size readings varied between $33 \mathrm{~nm}$ and $85 \mathrm{~nm}$ with a polydispersity of 0.3 (moderate dispersion). The size range seemed to be suited for fluorescence quenching of E2 molecules that were nonspecifically bound to MIP particles.

Figure 6 plots the fluorescence quenching efficiency $\left(\theta=1-F / F_{0}\right.$, where $F$ and $F_{0}$ are the fluorescence emission intensities measured in the presence and absence of quencher) versus the concentration of quencher. With AuNPs, similar quenching efficiencies were observed for both particles and E2 in (a), (b), and (c). By comparison, sodium nitrite exhibited significantly lower quenching efficiency in (d) and (e). Approximately $4500 \mathrm{ppm}$ sodium nitrite was needed in (e) to quench $80 \%$ of fluorescence emission from E2-MIP particles although only $38.7 \mathrm{ppm}$ AuNPs was needed in (b). Interestingly a lesser amount of sodium nitrite was needed in the presence of AuNPs in (d) to quench E2-MIP particles, from $80 \%$ to $90 \%$, than in (e).

3.3. Stern-Volmer Plots. All fluorescence quenching data were analyzed further by applying the Stern-Volmer (S$\mathrm{V})$ equations that examine different quenching mechanisms $[18,22]$ :

$$
\begin{gathered}
\frac{F_{0}}{F}=1+K_{\mathrm{sv}}[Q], \\
\frac{F_{0}}{F}=\left(1+K_{\mathrm{sv}}[Q]\right) \exp (V[Q]) .
\end{gathered}
$$

$F_{0}$ and $F$ are the fluorescence emission intensities in the absence and presence of quencher. $K_{s v}$ denotes the dynamic

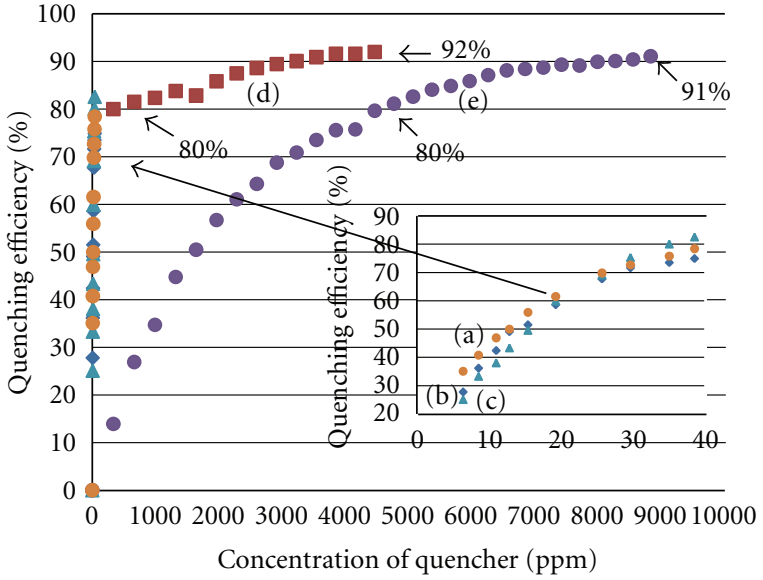

Figure 6: Quenching efficiency $(\theta)$ versus concentration of quencher: (a) $2.5 \mathrm{mg} / \mathrm{mL}$ E2-bound NIP particles in aqueous suspension quenched with AuNPs, (b) $2.5 \mathrm{mg} / \mathrm{mL}$ E2-MIP particles in aqueous suspension quenched with AuNPs, (c) $3.5 \mathrm{ppm}$ E2 aqueous solution quenched with AuNPs, and (d) $2.5 \mathrm{mg} / \mathrm{mL}$ E2MIP particles in aqueous suspension quenched first with AuNPs and next with sodium nitrite. (e) $2.5 \mathrm{mg} / \mathrm{mL}$ E2-MIP particles in aqueous suspension quenched with sodium nitrite (up to $0.128 \mathrm{M}$ ).

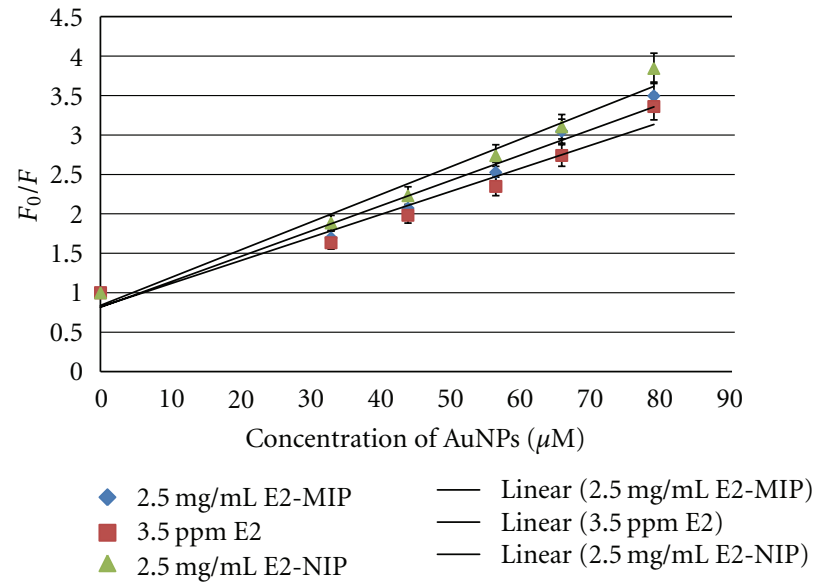

Figure 7: Stern-Volmer plots of $F_{0} / F$ versus concentration of AuNPs for $3.5 \mathrm{ppm}$ E2 aqueous solution, $2.5 \mathrm{mg} / \mathrm{mL}$ E2-MIP particles in aqueous suspension, and $2.5 \mathrm{mg} / \mathrm{mL}$ E2-bound NIP particles in aqueous suspension. Each solid line indicates the best possible linear regression.

quenching constant, and $V$ denotes the static quenching constant. $[Q]$ is the concentration of quencher. Equation (1) represents a linear function between dynamic quenching and quencher concentration, where quencher collision with the excited fluorophore $(\mathrm{E} 2 *)$ returns it to the ground state without fluorescence emission [23]. Figure 7 shows the linear S-V plots for AuNPs, which were best analyzed using (1). The $K_{\mathrm{sv}}$ for E2 is $2.6( \pm 0.1) \times 10^{4} \mathrm{M}^{-1}\left(R^{2}=0.9478\right)$, the $K_{\mathrm{sv}}$ for E2-MIP particles is $2.9( \pm 0.1) \times 10^{4} \mathrm{M}^{-1}\left(R^{2}=\right.$ $0.9566)$, and the $K_{\mathrm{sv}}$ for E2-bound NIP particles is $3.3( \pm 0.1)$ $\times 10^{4} \mathrm{M}^{-1}\left(R^{2}=0.9678\right)$. Due to their large size, AuNPs could hardly penetrate the porous structures of E2-MIP and 


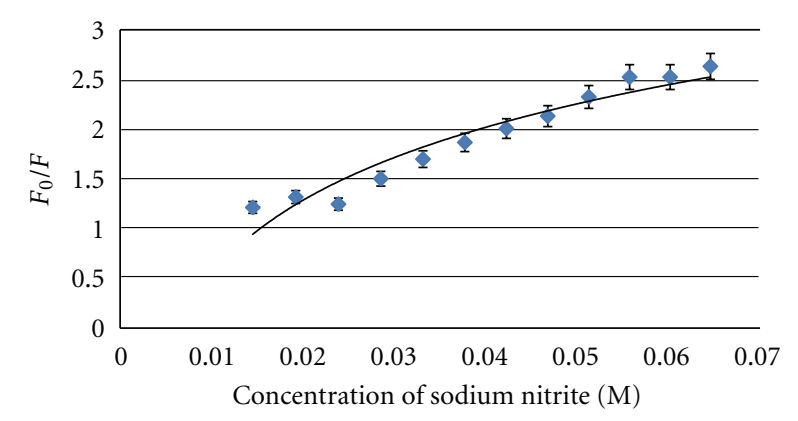

FIGURE 8: Stern-Volmer plots of $F_{0} / F$ versus concentration of sodium nitrite. Sodium nitrite was titrated stepwise into $2.5 \mathrm{mg} / \mathrm{mL}$ E2-MIPs in aqueous suspension containing $5.88 \times 10^{-4} \mathrm{M}$ AuNPs. Each solid line indicates the best possible regression.

E2-NIP particles. Consequently, their $K_{\mathrm{sv}}$ values were in the same order of magnitude as that obtained for E2 in aqueous solution.

The S-V plots for sodium nitrite was found to be nonlinear (with an upward-curving trend), as shown in Figure 8. Nonlinear S-V plots had been discussed by Zhao and Swager [22] as a combined result from dynamic and static quenchings. In contrast to dynamic quenching, the mechanism of static quenching involves interaction between the quencher and fluorophore to form a nonfluorescent complex [23]. Analysis using (2) obtained $K_{\mathrm{sv}}=2.1 \times$ $10^{2} \mathrm{M}^{-1}$ and static quenching constant $(V)$ below $1.0 \mathrm{M}^{-1}$ $\left(R^{2}=0.9220\right)$ for $2.5 \mathrm{mg} / \mathrm{mL}$ E2-MIPs in aqueous suspension containing $5.88 \times 10^{-4} \mathrm{M}$ AuNPs. Since MIP cavities did not facilitate complex formation between E2 molecules and nitrite anions due to space constraints, the $V$ value turned out to be very small. A higher $V$ value of $4.7 \mathrm{M}^{-1}$ was obtained for 4.5 ppm E2 in aqueous solution, which signifies the complexation of E2 molecules with nitrite anions in the absence of steric hindrance.

3.4. Determination of E2 in Water. Various concentrations (from $0.1 \mathrm{ppb}$ up to $3.5 \mathrm{ppm}$ ) of E2 in aqueous solution were used to validate MIP particles for rapid E2 determination by fluorescence quenching. MIP particles were added in these E2 solutions to form $0.5-3.5 \mathrm{mg} / \mathrm{mL}$ suspensions. Two incubation times ( $5 \mathrm{~min}$ and $35 \mathrm{~min}$ ) were tested to investigate binding equilibrium between E2 molecules and MIP particles. The fluorescence emission intensity from E2 specifically bound with MIP particles was determined by measuring the fluorescence emission intensities after two quenching steps, as summarized by

$$
\begin{aligned}
I_{\mathrm{E} 2 \text { inside MIP cavities }}= & I_{\mathrm{After}} \text { quenching with AuNps } \\
& -I_{\mathrm{After}} \text { quenching with sodium nitrite }
\end{aligned}
$$

Essentially, the first quenching with AuNPs would eliminate the fluorescence emission from E2 molecules on nonspecific binding sites throughout the porous MIP particles. Then, sodium nitrite would quench the fluorescence emission from E2 molecules inside the specific imprinted

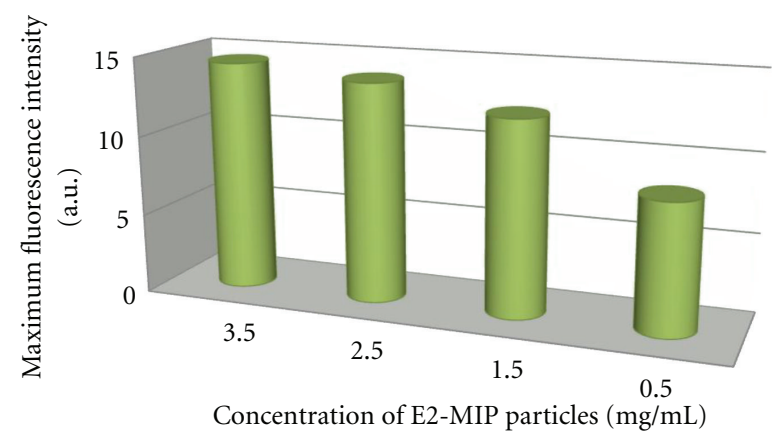

Figure 9: $I_{\max }$ for $3.5 \mathrm{mg} / \mathrm{mL}, \quad 2.5 \mathrm{mg} / \mathrm{mL}, \quad 1.5 \mathrm{mg} / \mathrm{mL}$, and $0.5 \mathrm{mg} / \mathrm{mL}$ E2-MIP particles in aqueous suspension.

cavities. The normalized \% binding of E2 with imprinted cavities was determined as

$$
\text { Normalized \% E2 binding }=\frac{I}{I_{\max }},
$$

where $I$ is the fluorescence intensity from E2 bound specifically inside imprinted cavities for an E2 standard solution, and $I_{\max }$ is the maximum fluorescence intensity from E2 bound specifically inside all imprinted cavities. Both $I$ and $I_{\max }$ were calculated according to (3), in parallel measurements. To determine $I_{\max }$, E2-MIP particles were prepared by washing only with DDW to remove all nonspecifically bound E2 molecules. Normalization was deemed necessary because $I_{\max }$ is not linearly dependent on the concentration of E2MIP particles in aqueous suspension, as shown in Figure 9, due to inner filter effects.

Figure 10 shows \% E2 binding (with imprinted cavities) as a function of E2 concentration after incubation for (a) $5 \mathrm{~min}$ and (b) $35 \mathrm{~min}$. A comparison of (a) with (b) indicates that \% E2 binding exhibited no significant difference. This suggests that binding equilibrium was reached in $5 \mathrm{~min}$ or less, in agreement with the $\sim 2$ min previously reported [24]. As $0.5 \mathrm{mg} / \mathrm{mL}$ E2-MIP particles had the smallest number of imprinted cavities and hence the lowest $I_{\max }$ value, it produced the highest \% E2 binding among the three concentrations studied. As the method involved binding of the analyte with MIP particles for the best possible selectivity (only second to natural antibodies), Scatchard plots were constructed in accordance with the following equation [25]:

$$
\% \text { E binding }=\frac{[\mathrm{E} 2]}{\left(K_{d}+[\mathrm{E} 2]\right)}
$$

where $K_{d}$ is the equilibrium binding constant. The best calibration curves, fitted using Graphpad Prism, are shown as solid lines in Figure 10. The best correlation coefficients were obtained for $2.5 \mathrm{mg} / \mathrm{mL}$ MIP particles, being $R^{2}=0.9716$ for $5 \mathrm{~min}$ of incubation and 0.9937 for $35 \mathrm{~min}$ of incubation. Table 1 shows that the equilibrium binding constant $\left(K_{d}\right)$ for $2.5 \mathrm{mg} / \mathrm{mL}$ of MIP particles, using $5 \mathrm{~min}$ of incubation, was the highest among the three MIP concentrations studied as expected. 
TABLE 1: Equilibrium binding constant $\left(K_{d}\right)$ values determined for three concentrations of MIP particles in aqueous suspension, after 5 min of incubation.

\begin{tabular}{lcc}
\hline $\begin{array}{l}\text { Incubation time } \\
(\mathrm{min})\end{array}$ & $\begin{array}{c}\text { Concentration of MIP particles } \\
(\mathrm{mg} / \mathrm{mL})\end{array}$ & $\begin{array}{c}K_{d} \\
(\mathrm{ppm})\end{array}$ \\
\hline \multirow{3}{*}{$5 \mathrm{~min}$} & 0.5 & 0.7 \\
& 1.5 & 1.4 \\
& 2.5 & 2.9 \\
\hline
\end{tabular}

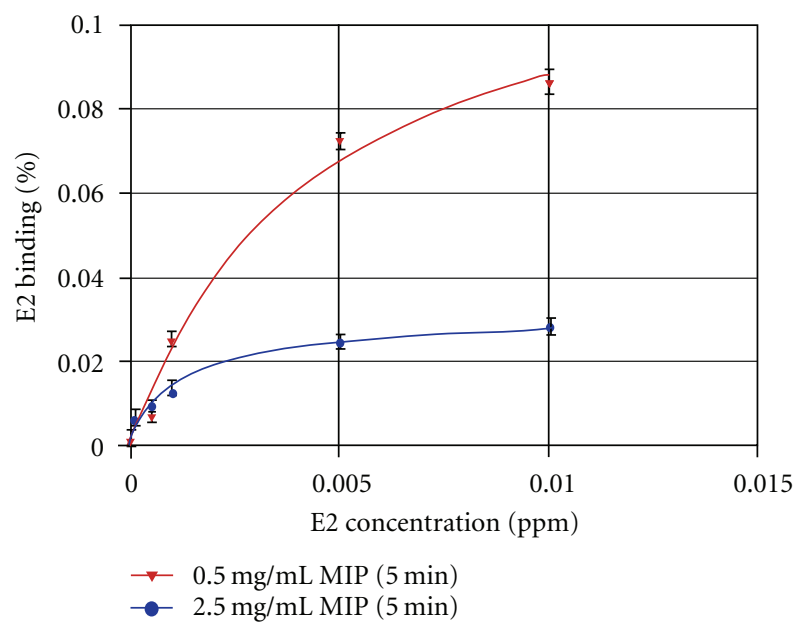

(a)

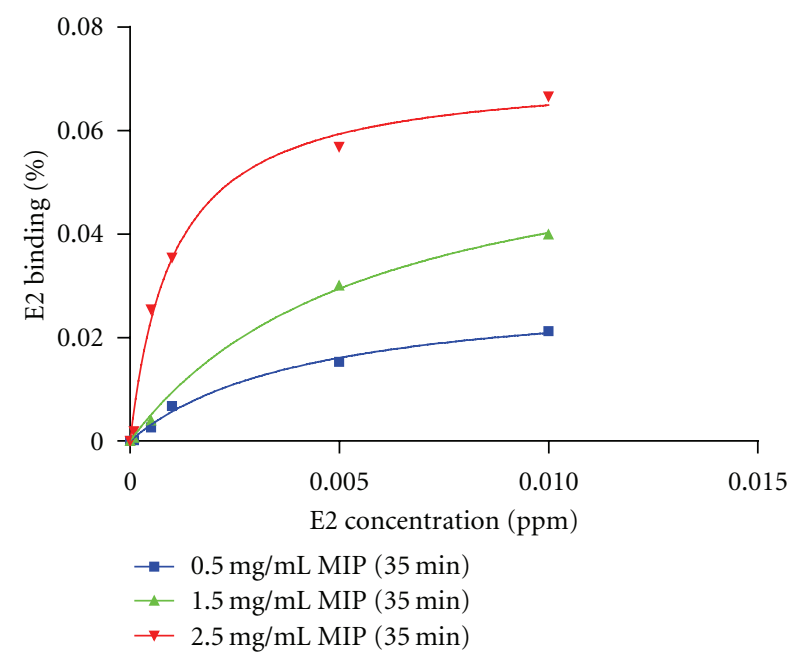

(b)

FIGURE 10: \% E2 binding (with imprinted cavities) as a function of E2 concentration after incubation for (a) $5 \mathrm{~min}$, and (b) $35 \mathrm{~min}$ (standard error bars, approximately three- to five-times the size of each data point symbol, are omitted here for the sake of clarity).

\section{Conclusion}

AuNPs $(43 \pm 5 \mathrm{~nm})$ were efficient in quenching the fluorescence emission from E2 molecules in aqueous solution, or nonspecifically bound with porous particles. For E2 molecules specifically bound inside imprinted cavities, fluorescence quenching by sodium nitrite was successful. A rapid method (under $10 \mathrm{~min}$ ) has been developed for E2 determination by measuring the change in fluorescence emission intensities between these two fluorescence quenching steps, using first AuNPs and then sodium nitrite. One major advantage of this method is the high selectivity of MIP particles for E2, as previously demonstrated using molecules with similar structures (estrone, ethynylestradiol) [21] and dissimilar structures (bisphenol A) [26]. Other fluorescent molecules would not interfere with the E2 determination because they can only bind nonspecifically to be readily quenched by AuNPs. Highly correlated Scatchard plots $\left(R^{2}>0.97\right)$ serve well as a standard calibration curve. The detection limit for E2 is low, at the ultratrace level of $0.1 \mathrm{ppb}$. The method is also promising for use on a portable spectrofluorometer in field studies. Further work is underway to verify that no potential interference by common organics and anions $\left(\mathrm{CO}_{3}^{2-}, \mathrm{NO}_{3}^{-}\right.$, $\mathrm{PtCl}_{4}^{2-}, \mathrm{SCN}^{-}, \mathrm{N}_{3}^{-}$present in environmental waters) exists after an extra centrifugation step is added (to precipitate the MIP particles out for transfer into a cuvette of deionized water) before the two fluorescence quenching measurements. Optimization of the method will also be completed with testing of real sample matrices from environmental waters.

\section{Acknowledgment}

Financial support of the Natural Sciences and Engineering Research Council (NSERC) Canada is gratefully acknowledged.

\section{References}

[1] H. Yan and H. R. Kyung, "Characteristic and synthetic approach of molecularly imprinted polymer," International Journal of Molecular Sciences, vol. 7, no. 5-6, pp. 155-178, 2006.

[2] W. H. Zhou, C. H. Lu, X. C. Guo, F. R. Chen, H. H. Yang, and X. R. Wang, "Mussel-inspired molecularly imprinted polymer coating superparamagnetic nanoparticles for protein recognition," Journal of Materials Chemistry, vol. 20, no. 5, pp. 880-883, 2010.

[3] N. D. Zakaria, N. A. Yusof, J. Haron, and A. H. Abdullah, "Synthesis and evaluation of a molecularly imprinted polymer for 2,4-Dinitrophenol," International Journal of Molecular Sciences, vol. 10, no. 1, pp. 354-365, 2009.

[4] P. Kueseng, M. L. Noir, B. Mattiasson, P. Thavarungkul, and P. Kanatharana, "Molecularly imprinted polymer for analysis of trace atrazine herbicide in water," Journal of Environmental Science and Health B, vol. 44, no. 8, pp. 772-780, 2009.

[5] K. Prasad, K. P. Prathish, J. M. Gladis, G. R. K. Naidu, and T. P. Rao, "Molecularly imprinted polymer (biomimetic) based potentiometric sensor for atrazine," Sensors and Actuators B, vol. 123, no. 1, pp. 65-70, 2007.

[6] K. P. Prathish, K. Prasad, T. P. Rao, and M. V. S. Suryanarayana, "Molecularly imprinted polymer-based potentiometric sensor for degradation product of chemical warfare agents. Part I. Methylphosphonic acid," Talanta, vol. 71, no. 5, pp. 19761980, 2007.

[7] T. Alizadeh and M. Akhoundian, "A novel potentiometric sensor for promethazine based on a molecularly imprinted polymer (MIP): the role of MIP structure on the sensor performance," Electrochimica Acta, vol. 55, no. 10, pp. 3477$3485,2010$. 
[8] J. Zhang, Y. Wang, R. Lv, and L. Xu, "Electrochemical tolazoline sensor based on gold nanoparticles and imprinted poly-o-aminothiophenol film," Electrochimica Acta, vol. 55, no. 12, pp. 4039-4044, 2010.

[9] A. K. Patel, P. S. Sharma, and B. B. Prasad, "Trace-level sensing of creatine in real sample using a zwitterionic molecularly imprinted polymer brush grafted to sol-gel modified graphite electrode," Thin Solid Films, vol. 518, no. 10, pp. 2847-2853, 2010.

[10] N. Wu, L. Feng, Y. Tan, and J. Hu, "An optical reflected device using a molecularly imprinted polymer film sensor," Analytica Chimica Acta, vol. 653, no. 1, pp. 103-108, 2009.

[11] B. K. Lavine, D. J. Westover, N. Kaval, N. Mirjankar, L. Oxenford, and G. K. Mwangi, "Swellable molecularly imprinted polyN-(N-propyl)acrylamide particles for detection of emerging organic contaminants using surface plasmon resonance spectroscopy," Talanta, vol. 72, no. 3, pp. 1042-1048, 2007.

[12] B. B. Prasad, M. P. Tiwari, R. Madhuri, and P. S. Sharma, "Development of a highly sensitive and selective hyphenated technique (molecularly imprinted micro-solid phase extraction fiber-molecularly imprinted polymer fiber sensor) for ultratrace analysis of folic acid," Analytica Chimica Acta, vol. 662, no. 1, pp. 14-22, 2010.

[13] Y. Yang and E. P. C. Lai, "An investigation of porous structure in molecularly imprinted polymer for sensor development: non-linear fluorescence quenching of $17 \beta$-estradiol bound inside MIP submicron particles by sodium nitrite and methacrylamide," Journal of Photochemistry and Photobiology A, vol. 213, no. 2-3, pp. 123-128, 2010.

[14] J. Zhang, R. Badugu, and J. R. Lakowicz, "Fluorescence quenching of CdTe nanocrystals by bound gold nanoparticles in aqueous solution," Plasmonics, vol. 3, no. 1, pp. 3-11, 2008.

[15] S. Y. Lim, J. H. Kim, J. S. Lee, and C. B. Park, "Gold nanoparticle enlargement coupled with fluorescence quenching for highly sensitive detection of analytes," Langmuir, vol. 25, no. 23, pp. 13302-13305, 2009.

[16] S. Mayilo, M. A. Kloster, M. Wunderlich et al., "Long-range fluorescence quenching by gold nanoparticles in a sandwich immunoassay for cardiac troponin T," Nano Letters, vol. 9, no. 12, pp. 4558-4563, 2009.

[17] S. Wei, A. Molinelli, and B. Mizaikoff, "Molecularly imprinted micro and nanospheres for the selective recognition of 17betaestradiol," Biosensors and Bioelectronics, vol. 21, no. 10, pp. 1943-1951, 2006.

[18] S. K. Ghosh, A. Pal, S. Kundu, S. Nath, and T. Pal, "Fluorescence quenching of 1-methylaminopyrene near gold nanoparticles: size regime dependence of the small metallic particles," Chemical Physics Letters, vol. 395, no. 4-6, pp. 366372, 2004.

[19] R. A. Leese, "Corrections for inner-filter effects in fluorescence quenching measurements via right-angle and front-surface illumination," Analytical Chemistry, vol. 50, no. 8, pp. 11931197, 1978.

[20] Z. B. Zhang and J. Y. Hu, "Selective removal of estrogenic compounds by molecular imprinted polymer (MIP)," Water Research, vol. 42, no. 15, pp. 4101-4108, 2008.

[21] Y. Yang, E. P. C. Lai, and M. Liu, "Spectroscopic analysis of poly (methacrylic acid-co-ethylene glycol dimethacrylate) submicron particles by fluorescence emission and light scattering upon binding with $17 \beta$-estradiol in water treatment," Open Colloid Science Journal, vol. 3, no. 8, pp. 1-8, 2010.
[22] D. Zhao and T. M. Swager, "Sensory responses in solution vs solid state: a fluorescence quenching study of poly(iptycenebutadiynylene)s," Macromolecules, vol. 38, no. 22, pp. 93779384, 2005.

[23] J. R. Lakowicz, Principles of Fluorescence Spectroscopy, Springer, New York, NY, USA, 3rd edition, 2006.

[24] E. P. C. Lai, Z. D. Maleki, and S. Wu, "Characterization of molecularly imprinted and nonimprinted polymer submicron particles specifically tailored for removal of trace $17 \beta$-estradiol in water treatment," Journal of Applied Polymer Science, vol. 116, no. 3, pp. 1499-1508, 2010.

[25] T. A. Sergeyeva, S. A. Piletsky, A. A. Brovko et al., "Conductimetric sensor for atrazine detection based on molecularly imprinted polymer membranes," Analyst, vol. 124, no. 3, pp. 331-334, 1999.

[26] Z. DeMaleki, E. P. C. Lai, and E. Dabek-Zlotorzynska, "Capillary electrophoresis characterization of molecularly imprinted polymer particles in fast binding with 17betaestradiol," Journal of Separation Science, vol. 33, no. 17-18, pp. 2796-2803, 2010. 


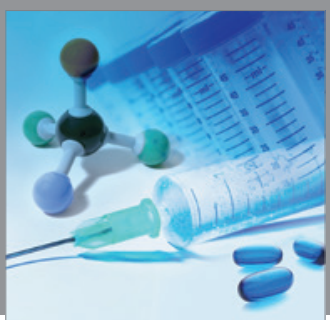

International Journal of

Medicinal Chemistry

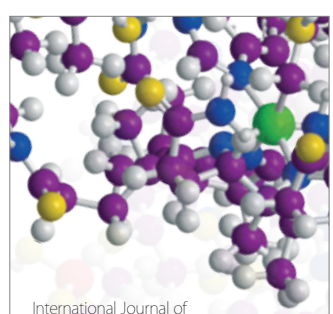

Carbohydrate Chemistry

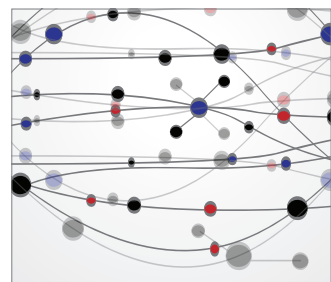

The Scientific World Journal
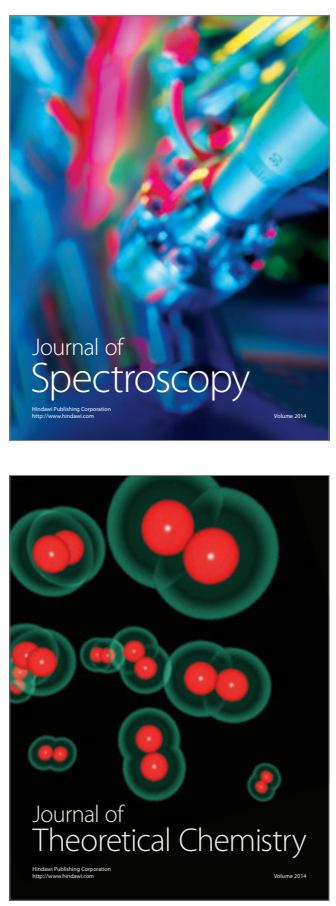
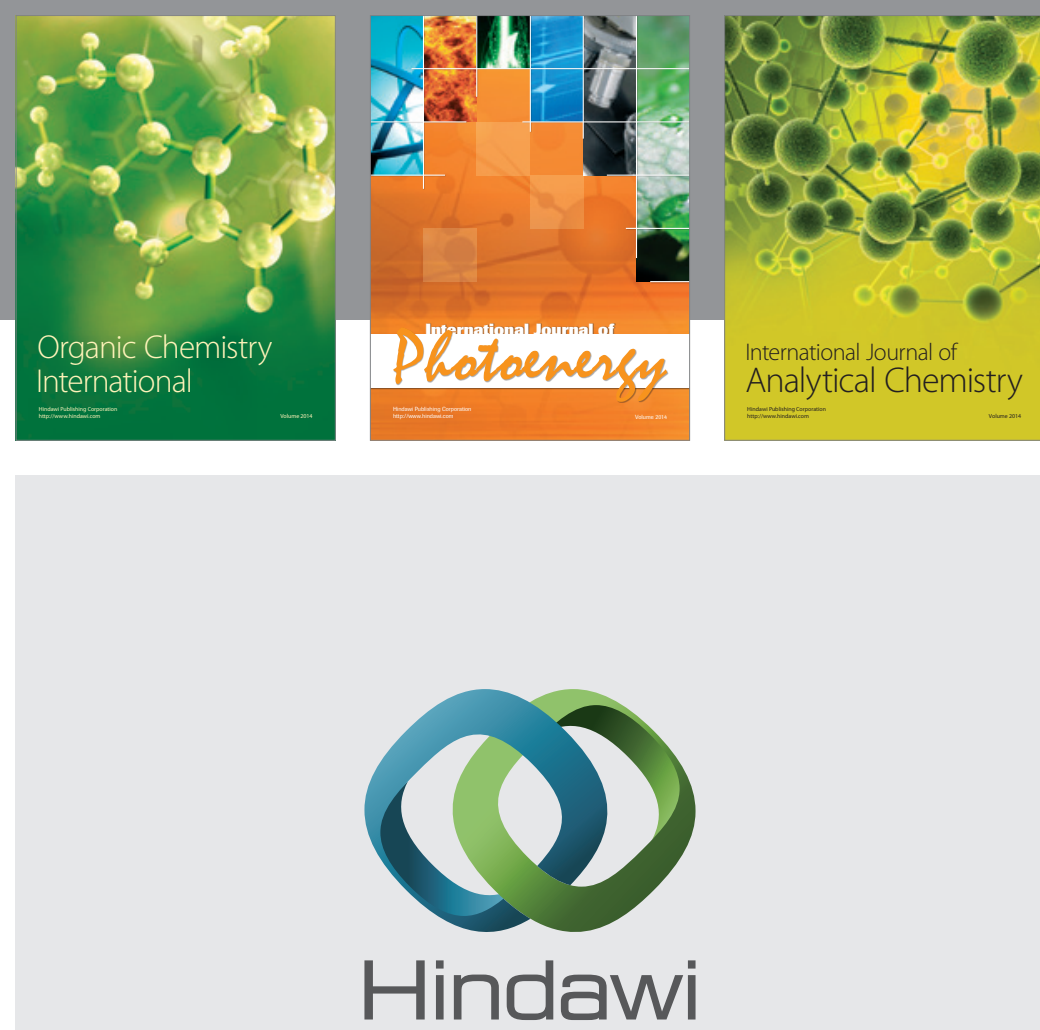

Submit your manuscripts at

http://www.hindawi.com
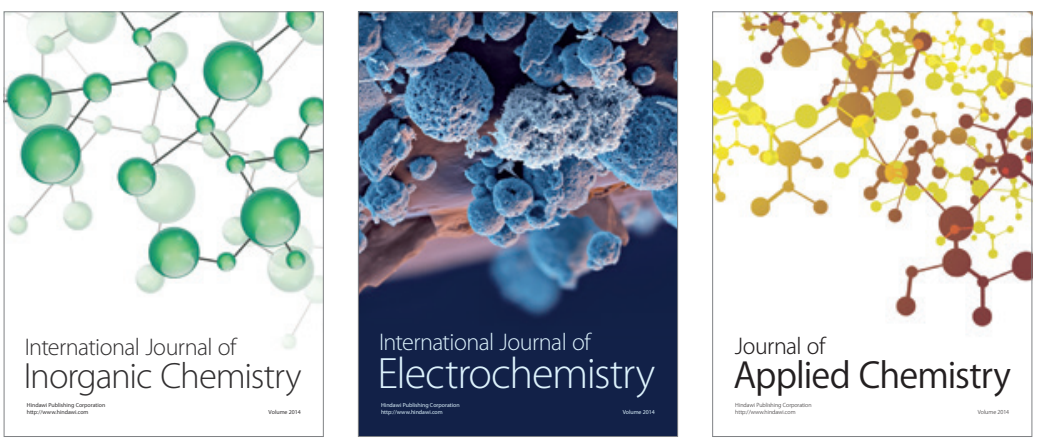

Journal of

Applied Chemistry
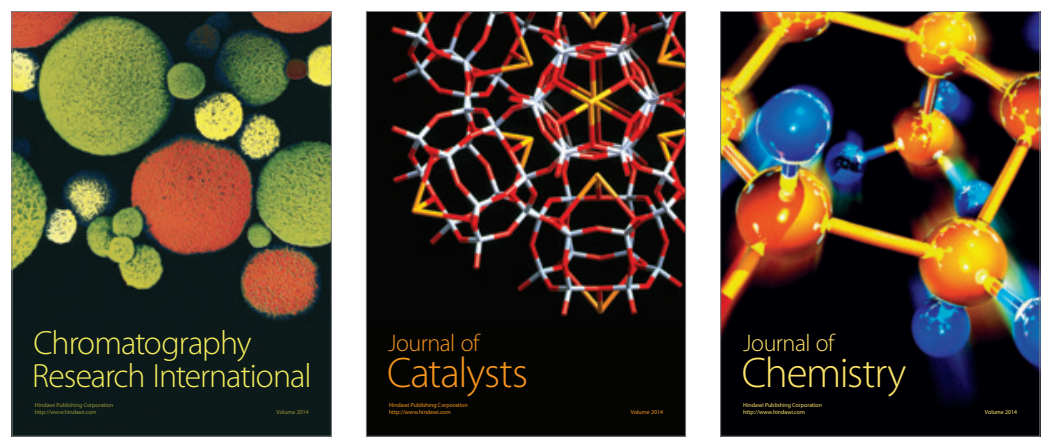
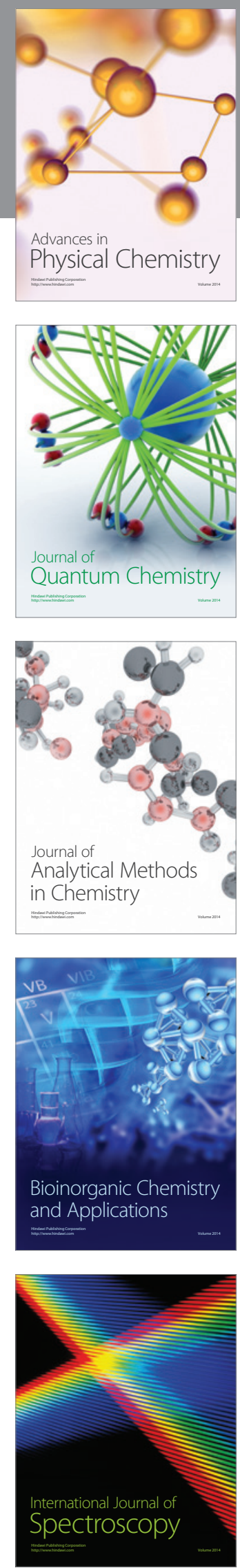